\title{
Composite Likelihood for Bilinear GARCH Model
}

\author{
Abdelhalim Bouchemella1, Fatima Zahra Benmostefa' \\ ${ }^{1}$ University of 08 May 1945, Guelma, Algeria \\ ${ }^{2}$ University of Badji Mokhtar, Annaba, Algeria \\ Email: abdelhalimgbs@yahoo.fr, benmostefafatima@yahoo.fr
}

Received 10 June 2014; revised 12 July 2014; accepted 20 July 2014

Copyright (C) 2014 by authors and Scientific Research Publishing Inc.

This work is licensed under the Creative Commons Attribution International License (CC BY).

http://creativecommons.org/licenses/by/4.0/

c) (i) Open Access

\begin{abstract}
In this study, we focus on the class of BL-GARCH models, which is initially introduced by Storti \& Vitale [1] in order to handle leverage effects and volatility clustering. First we illustrate some properties of BL-GARCH $(1,2)$ model, like the positivity, stationarity and marginal distribution; then we study the statistical inference, apply the composite likelihood on panel of BL-GARCH $(1,2)$ model, and study the asymptotic behavior of the estimators, like the consistency property and the asymptotic normality.
\end{abstract}

\section{Keywords}

Random Coefficient Autoregressive Model, BL-GARCH Models, Composite Likelihood

\section{Introduction}

Classical modelling of time series is not usually appropriate for financial data. Such as ARMA models do not allow the variability in volatility over time, are not able to capture asymmetries in the conditional variance of financial time series, and fail to generate the squared autocorrelations. In front of these monetary and financial problems, Engle [2] proposed a new class of autoregressive conditionally heteroscedastic models (ARCH), followed by generalized ARCH or GARCH suggested by Bollerslev [3]. Storti and Vitale [1] proposed an innovative approach to modelling leverage effects in financial time series based on the Bilinear GARCH noted by BL-GARCH models which are considered as a generalization of GARCH models.

In this present paper we study the BL-GARCH models, specifically BL-GARCH $(1,2)$ that is widely used and proved its performance for the volatility analysis of financial time series. We focus on studies of Storti \& Vitale [1] and Diongue \& Guégan and Wolff [4], which they have well discussed and treated this class of models. 
In recent years, several authors have been interested in composite maximum likelihood methods, that are widely used in parametric statistical inference, because of the good asymptotic properties of the estimators. The aim of composite likelihood is to reduce and simplify the computational complexity to cope with large datasets and presence of complex interdependencies.

The term pseudo-likelihood was originally proposed by Besag [5]. Lindsay [6] used the term composite likelihood for justify his choice to describe the method of construction considered. There are many research and studies in various fields, which have applied this method, for example in statistical genetics: Larribe and Fearnhead [7]; in time series: Richard, Davis and Chun Yip Yau [8] and Pakel, Shephard and Sheppard [9]; in longitudinal data: Molenberghs and Verbeke [10].

We organize this work as follows. In Section 2 we study the important properties of BL-GARCH $(1,2)$ concerning conditions for the positivity of conditional variance, conditions of stationarity and we conclude this section by the properties of marginal distribution. In Section 3 we introduce the BL-GARCH $(1,2)$ panel model, then we illustrate the good performance of estimators of composite likelihood applied to this model.

\section{Properties of BL-GARCH $(1,2)$}

We consider the asset log-returns $y_{t}$ at time $t$, assuming that

$$
\begin{gathered}
y_{t}=\mu_{t}+u_{t} \text { where } \mu_{t}=E\left(y_{t} / \Omega_{t-1}\right) \\
u_{t}=h_{t} \varepsilon_{t} \\
h_{t}^{2}=a_{0}+a_{1} u_{t-1}^{2}+b_{1} h_{t-1}^{2}+b_{2} h_{t-2}^{2}+c_{1} u_{t-1} h_{t-1}
\end{gathered}
$$

where $\Omega_{t-1}$ is the historical information set up to time $t-1$.

\subsection{Positivity of Conditional Variance}

We can write the model (2.3) in matrix form as:

$$
h_{t}^{2}=\left[1, u_{t-1}, h_{t-1}, h_{t-2}\right]\left[\begin{array}{cccc}
a_{0} & 0 & 0 & 0 \\
0 & a_{1} & \frac{1}{2} c_{1} & 0 \\
0 & \frac{1}{2} c_{1} & b_{1} & 0 \\
0 & 0 & 0 & b_{2}
\end{array}\right]\left[\begin{array}{c}
1 \\
u_{t-1} \\
h_{t-1} \\
h_{t-2}
\end{array}\right]=K_{t}^{\prime} A K_{t}
$$

Proposition 1. A sufficient set of conditions for positivity of conditional variance $h_{t}^{2}$ is

$$
a_{0}>0 ; \quad a_{1}>0 ; \quad b_{1}>0 ; \quad b_{2}>0 ; \quad c_{1}^{2}>4 a_{1} b_{1}
$$

Proof. We note that $h_{t}^{2}>0$ if and only if $A$ is a positive definite matrix, and this implies that all eigenvalues of $A$ are strictly positive.

Set of these eigenvalues are:

$$
\left\{a_{0} ; b_{2} ; \frac{1}{2}\left(a_{1}+b_{1}-\sqrt{a_{1}^{2}-2 a_{1} b_{1}+b_{1}^{2}+c_{1}^{2}}\right) ; \frac{1}{2}\left(a_{1}+b_{1}+\sqrt{a_{1}^{2}-2 a_{1} b_{1}+b_{1}^{2}+c_{1}^{2}}\right)\right\}
$$

\subsection{Stationarity}

We can rewrite the BL-GARCH $(1,2)$ as:

$$
\begin{gathered}
h_{t}^{2}=a_{0}+\left(a_{1} \varepsilon_{t-1}^{2}+b_{1}+c_{1} \varepsilon_{t-1}\right) h_{t-1}^{2}+b_{2} h_{t-2}^{2} \\
h_{t}^{2}=g\left(\varepsilon_{t-1}\right)+c\left(\varepsilon_{t-1}\right) h_{t-1}^{2}+d\left(\varepsilon_{t-1}\right) h_{t-2}^{2}
\end{gathered}
$$

which is a random coefficient autoregressive model of second order [RCAR(2)].

We put: $h_{t}^{2}=X_{t} ; g\left(\varepsilon_{t-1}\right)=e_{t} ; \quad c\left(\varepsilon_{t-1}\right)=\phi_{1}$ and $d\left(\varepsilon_{t-1}\right)=\phi_{2}$, we have

$$
X_{t}=\phi_{1} X_{t-1}+\phi_{2} X_{t-2}+e_{t}
$$




$$
\begin{aligned}
& \left(\begin{array}{c}
X_{t} \\
X_{t-1}
\end{array}\right)=\left(\begin{array}{cc}
\phi_{1} & \phi_{2} \\
1 & 0
\end{array}\right)\left(\begin{array}{c}
X_{t-1} \\
X_{t-2}
\end{array}\right)+\left(\begin{array}{c}
e_{t} \\
0
\end{array}\right) \\
& \underline{X}_{t}=\Phi \underline{X}_{t-1}+\underline{e}_{t} \\
& V_{t}=a_{0}\left[I_{2}-\Psi B\right]^{-1}
\end{aligned}
$$

with $E\left(\underline{X}_{t}\right)=V_{t}, E(\Phi)=\Psi$, and $B$ is backward operator. This implies that its eigenvalues are in the unit cercle.

So in order to the process be second order stationariry if and only if all eigenvalues are within unit cercle.

We can also rewrite the BL-GARCH $(1,2)$ :

$$
\begin{gathered}
\underline{Z}_{t}=\underline{b}_{t}+A_{t} \underline{Z}_{t-1} \\
{\left[\begin{array}{c}
u_{t}^{2} \\
h_{t}^{2} \\
h_{t-1}^{2} \\
u_{t} h_{t}
\end{array}\right]=\left[\begin{array}{c}
a_{0} \varepsilon_{t} \\
a_{0} \\
0 \\
a_{0}
\end{array}\right]+\left[\begin{array}{cccc}
a_{1} \varepsilon_{t}^{2} & b_{1} \varepsilon_{t}^{2} & b_{2} \varepsilon_{t}^{2} & c_{1} \varepsilon_{t}^{2} \\
a_{1} & b_{1} & b_{2} & c_{1} \\
0 & 1 & 0 & 0 \\
a_{1} \varepsilon_{t} & b_{1} \varepsilon_{t} & b_{2} \varepsilon_{t} & c_{1} \varepsilon_{t}
\end{array}\right]\left[\begin{array}{c}
u_{t-1}^{2} \\
h_{t-1}^{2} \\
h_{t-2}^{2} \\
u_{t-1} h_{t-1}
\end{array}\right]}
\end{gathered}
$$

Remark 2. 1) $A_{t}$ is $(p+q+r) \times(p+q+r)$ matrix and $r=\min (p, q)$ in general case of BL-GARCH $(p, q)$ model.

2) Equation (2.8) is random coefficient $\operatorname{VAR}(1)$.

3) $\left(\underline{Z}_{t}\right)_{t \geq 1}$ is a Markov process.

Theorem 3. (strict stationarity)

In order to exist a strict stationary solution of Equation (2.8) it is necessary and sufficient that

$$
\gamma<0
$$

where $\gamma=\lim _{t \rightarrow+\infty} \frac{1}{t} \log \left\|A_{t} A_{t-1} \cdots A_{1}\right\|$, is the largest Lyapunov exponent of the model (2.8).

If this solution exists, then it is unique strictly stationary,non anticipative and ergodic.

Proof. See [11].

Example 4. For the BL-GARCH $(1,2)$ we consider the matrix

$$
A_{t}=\left[\begin{array}{cccc}
a_{1} \varepsilon_{t}^{2} & b_{1} \varepsilon_{t}^{2} & b_{2} \varepsilon_{t}^{2} & c_{1} \varepsilon_{t}^{2} \\
a_{1} & b_{1} & b_{2} & c_{1} \\
0 & 1 & 0 & 0 \\
a_{1} \varepsilon_{t} & b_{1} \varepsilon_{t} & b_{2} \varepsilon_{t} & c_{1} \varepsilon_{t}
\end{array}\right]
$$

for some values attributed to the coefficients $a_{1}, b_{1}, b_{2}, c_{1}$, we can simulate $\hat{\gamma}$ and $\varepsilon_{t} \sim N(0,1)$ or $\varepsilon_{t} \sim t_{n}$.

Estimation of $\gamma$ from 1000 simulations of size $t=1000$.

This simulation does know us the region of stationarity of BL-GARCH $(1,2)$.

If $a_{1}+b_{1}+b_{2}=1$, there is no stationary solution (strict or 2 nd order), while there is a strictly stationary solution of an IGARCH model under general conditions.

\subsection{Marginal Distribution}

From (2.8) and by recursive, we have:

$$
\underline{Z}_{t}=\underline{b}_{t}+\sum_{k=1}^{+\infty} A_{t} A_{t-1} \cdots A_{t-k+1} \underline{b}_{t-k}
$$

we put, for $k>0$,

$$
A_{t, k}=A_{t} A_{t-1} \cdots A_{t-k+1} \text { and } \underline{Z}_{t, k}=A_{t, k} \underline{b}_{t-k}
$$

we denote $\otimes$ the Kronecker product and $\|\cdot\|$ matrix norm, then 


$$
E\left\|\underline{Z}_{t, k}\right\|^{m}=E\left\|A_{t, k}^{\otimes m} \underline{\underline{b}}_{t-k}^{\otimes m}\right\|=E\left\|A_{t}^{\otimes m} A_{t-1}^{\otimes m} \cdots A_{t-k+1}^{\otimes m} \underline{b}_{t-k}^{\otimes m}\right\|=\left\|A^{(m)} \underline{b}^{(m)}\right\|
$$

using product matricies independence $A_{t} A_{t-1} \cdots A_{t-k+1} \underline{b}_{t-k}$, because $\left(\varepsilon_{t}\right)$ are iid, we have

$$
\left\|\underline{Z}_{t}\right\|_{m}=\left[E\left\|\underline{Z}_{t}\right\|^{m}\right]^{1 / m}=\sum_{k=0}^{\infty}\left\|\underline{Z}_{t, k}\right\|_{m} \leq\left\{\sum_{k=0}^{\infty}\left\|\left(A^{(m)}\right)^{k}\right\|^{1 / m}\right\}\left\|\underline{b}^{(m)}\right\|^{1 / m}
$$

If the spectral radius $\rho\left(A^{(m)}\right)<1$ of the matrix $A^{(m)}$, then $\left\|\left(A^{(m)}\right)^{k}\right\| \rightarrow 0$ as $k \rightarrow+\infty$, thus $\left\|u_{t}\right\|_{m} \leq\left\|\underline{Z}_{t}\right\|_{m}$. So $\rho\left(A^{(m)}\right)<1$ is sufficient condition for the exisrence of $E\left(u_{t}^{2 m}\right)$. For more details, it is recommended to refer to [11].

Theorem 5. Suppose that $E\left(\varepsilon_{t}^{2 m}\right)$ and $\rho\left(A^{(m)}\right)<1$, then for each $t \varepsilon \mathbb{Z},\left(\underline{Z}_{t}\right)_{t}$ defined by (2.8) converge in $L^{m}$ and the process $\left(u_{t}^{2}\right)_{t}$ defined as the first component of $\underline{Z}_{t}$ is $2 m$-order strictly stationary solution.

We make a simulation given some values of coefficients and a distribution of $\varepsilon_{t}$ in order to calculate $\rho\left(A^{(m)}\right), E\left(\varepsilon_{t}^{2 m}\right)$, and $E\left(u_{t}^{2 m}\right)$.

\section{The BL-GARCH Panel}

We assumed that we have panel of asset returns, with $T$ observations and $N$ assets. The return on asset $i$ at time $t$ is $y_{i t}$, where $i=1, \cdots, N$ and $t=1, \cdots, T$, given by:

$$
\begin{aligned}
& y_{i t}=\mu_{i t}+u_{i t} \\
& u_{i t}=h_{i t} \varepsilon_{i t} \\
& h_{i t}^{2}=a_{0 i}\left(1-a_{1}-b_{1}-b_{2}\right)+a_{1} u_{i t-1}^{2}+b_{1} h_{i t-1}^{2}+b_{2} h_{i t-2}^{2}+c_{1} u_{i t-1} h_{i t-1}
\end{aligned}
$$

where $a_{0 i}>0, a_{1}+b_{1}+b_{2}<1$ and $a_{1}, b_{1}, b_{2} \in\left[0,1\left[\right.\right.$ with $c_{1}>2 a_{1} b_{1}$.

We consider $a_{0 i}$ as a nuisance parameters, and $\theta=\left(a_{1}, b_{1}, b_{2}, c_{1}\right)$ as a vector of interest parameters. Using the covariance tracking, suggested by Engle and Mezrich [12], we have:

$$
E\left(y_{i t}^{2}\right)=a_{0 i}
$$

then we can use the method of moment to estimate the nuisance parameter. Assuming a stochastic independence over $i$ and $t$, then the maximum likelihood estimator of $\theta$ is typically inconsistent for finite $T$, and $N \rightarrow \infty$. In order to overcome this problem and get a consistent estimator Engle, Shephard and Sheppard [13] allowed that $T$ to be large, and $N$ relates to $T$ and reduce the rate of convergence to $\sqrt{T}$ not $\sqrt{N T}$, noted in [13], followed by the same study and consideration of Pakel, Shephard and Sheppard [9].

\subsection{Composite Maximum Likelihood}

In this subsection we apply composite maximum likelihood method, that is widely used in time series in place of full likelihood when for example we want to reduce the computational complexity, or make inference about parameters of interest without making assumptions on the whole joint distribution of the data.

Given the data $y=\left(y_{1}, y_{2}, \cdots, y_{T}\right)$ where $y_{t}=\left(y_{1 t}, y_{2 t}, \cdots, y_{N t}\right)$ and let $f\left(y_{i t} / \Omega_{i, t-1}\right)$ be the conditional density for $y_{i t}$, we put $\phi_{i}=\left(a_{i 0}, \theta\right)$ and $\phi_{(N)}=\left(\phi_{1}, \phi_{2}, \cdots, \phi_{N}\right)$. Our estimation procedure focuses on two-step. We begin by application of moment method to estimate nuisance parameters using (3.2), then we apply composite likelihood to estimate $\theta$ which is defined by:

$$
C L\left(y, \phi_{(N)}\right)=\frac{1}{T} \sum_{t=1}^{T}\left[\frac{1}{N} \sum_{i=1}^{N} \log f\left(y_{i t} / \Omega_{i, t-1} ; \phi_{i}\right)\right]
$$

In our situation we use the variation-free as Engle, Shephard and Sheppard [13] and Engle, Hendry and Richard [14], then we obtain the composite maximum likelihood estimator by solving 


$$
\hat{\theta}_{C L}=\arg \max _{\theta} \frac{1}{T} \sum_{t=1}^{T} \frac{1}{N} \sum_{i=1}^{N} \log f\left(y_{i t} / \Omega_{i, t-1} ; \hat{a}_{i 0}, \theta\right)
$$

where $\hat{a}_{i 0}$ for each $i$ is obtained by solving

$$
\sum_{t=1}^{T} Q_{i t}\left(\theta, \hat{a}_{i 0}\right)=0
$$

From (3.2) we have

$$
\begin{gathered}
Q_{i t}\left(\theta, a_{i 0}\right)=y_{i t}^{2}-a_{0 i} ; \quad E\left(Q_{i t}\left(\theta, a_{i 0}^{*}\right)\right)=0 \\
\frac{1}{T} \sum_{t=1}^{T} Q_{i t}\left(\theta, \hat{a}_{i 0}\right)=0
\end{gathered}
$$

where $a_{i 0}^{*}$ is the true value of $a_{i 0}$ for each $i$. Stacking (3.5) for $i=1, \cdots, N$, we have

$$
Q_{(N)}\left(y_{t}, \phi_{(N)}\right)=\left[\begin{array}{c}
y_{1 t}^{2}-a_{01} \\
\vdots \\
y_{N t}^{2}-a_{0 N}
\end{array}\right] \Rightarrow E\left(Q_{(N)}\left(y_{t}, \phi_{(N)}^{*}\right)\right)=0
$$

On the other hand, for the interest parameter $\theta$, we use composite likelihood, considering the following three typical distributions:

1) The score function for the normal density composite likelihood function is:

$$
W_{1}\left(y_{t}, \theta, \phi_{(N)}\right)=\frac{\partial}{\partial \theta} \frac{1}{N}\left(-\frac{1}{2} \sum_{i=1}^{N} \log h_{i t}^{2}-\frac{1}{2} \sum_{i=1}^{N} \frac{u_{i t}^{2}}{h_{i t}^{2}}\right)
$$

2) The score function for the cauchy density composite likelihood function is:

$$
W_{2}\left(y_{t}, \theta, \phi_{(N)}\right)=\frac{\partial}{\partial \theta}\left(-N \log \pi+\sum_{i=1}^{N} \log h_{i t}-\sum_{i=1}^{N} \log \left(h_{i t}^{2}+u_{i t}^{2}\right)\right)
$$

3) The score function for the student density composite likelihood function is:

$$
W_{3}\left(y_{t}, \theta, \phi_{(N)}\right)=\frac{\partial}{\partial \theta} N\left(\log \Gamma\left(\frac{v+1}{2}\right)-\log \Gamma\left(\frac{v}{2}\right)-h_{i t}^{2}-\frac{1}{2} \sum_{i=1}^{N} \frac{u_{i t}^{2}}{h_{i t}^{2}}\right)
$$

For $i=1,2,3$ we put:

$$
W_{i}\left(y_{t}, \theta, \hat{\phi}_{(N)}\right)=0
$$

where $\hat{\phi}_{(N)}$ is a moment estimator.

The sample moment conditions for each of (3.8), (3.9) and (3.10) are given by:

$$
\frac{1}{T} \sum_{t=1}^{T} W_{i}\left(y_{t}, \hat{\theta}, \hat{\phi}_{(N)}\right)=0 ; \text { for } i=1,2,3
$$

We put:

$$
K_{i}\left(y_{t}, \theta^{*}, \phi_{(N)}^{*}\right)=\left[\begin{array}{c}
Q_{(N)}\left(y_{t}, \phi_{(N)}^{*}\right) \\
W_{i}\left(y_{t}, \theta^{*}, \phi_{(N)}^{*}\right)
\end{array}\right]
$$

then we imply that:

$$
E\left[K_{i}\left(y_{t}, \theta^{*}, \phi_{(N)}^{*}\right)\right]=0 \text { and } \frac{1}{T} \sum_{t=1}^{T} K_{i}\left(y_{t}, \hat{\theta}, \hat{\phi}_{(N)}\right)=0 ; \text { for } i=1,2,3
$$

(3.6) and (3.11) are the first order condition for the maximisation problem of (3.4). 


\subsection{Asymptotic Behavior}

In this subsection we attempt to obtain the asymptotic properties of composite likelihood estimator, based on a reasonable initial moment estimator for nuisance parameters. We show under which initial conditions to have a consistent estimator and asymptotic normality with the standard root- $T$ convergence rate and $N$ can potentially increase with $T$.

Engle, Shephard and Sheppard [13] have obtained consistency property and central limit theorem for $\hat{\theta}_{C L}$ under some regularity conditions, and also Billy Wu, Qiwei Yao and Shiwu Zhu [15]. Through the following two fundamental theorems, we will show the consistency and central limit theorem for $\hat{\theta}_{C L}$ when $T \rightarrow \infty$ while $N$ can potentially increase with $T$.

Theorem 6. We consider the following assumptions:

1) The condition (3.5) holds.

2) We assume that the parameters spaces are compacts.

3) Suppose that

$$
\operatorname{argmax} \frac{1}{T N} \sum_{t=1}^{T} \sum_{i=1}^{N} \log f\left(y_{i t} / \Omega_{i, t-1} ; a_{i 0}^{*}, \theta\right) \underline{p} \theta^{*},
$$

4) $\log f\left(y_{i t} / \Omega_{i, t-1} ; a_{i 0}^{*}, \theta\right)$ is continuously differentiable in $a_{i 0}$.

5) Assume that the following sum satisfies a weak law large number as $T \rightarrow \infty$

$$
\frac{1}{T} \sum_{t=1}^{T} \frac{1}{N} \sum_{i=1}^{N} \sup _{i 0}, \theta\left|\frac{\partial \log f\left(y_{i t} / \Omega_{i, t-1} ; a_{i 0}, \theta\right)}{\partial a_{i 0}}\right|,
$$

6) Assume that

$$
\sup _{\theta} \max _{i \in\{1, \cdots, N\}}\left|\hat{a}_{i 0}-a_{i 0}\right| \underline{p} 0,
$$

then there exists a solution of the likelihood Equation (3.11), for which $\hat{\theta} \underline{p} \theta^{*}$.

Proof. See [13].

Theorem 7. For any consistent solution of the likelihood Equation (3.11), we assume that:

1) $Q_{i t}\left(\theta, a_{i 0}\right)$ is once continuously differentiable.

2) $\left(a_{i 0}^{*}, \theta^{*}\right)$ is an interior point of $\left(\Lambda_{i} \times \Theta\right)$.

3) We put

$$
\begin{aligned}
& Y_{t, T}=\frac{1}{N} \sum_{i=1}^{N}\left[\frac{\partial \log f\left(y_{i t} / \Omega_{i, t-1} ; a_{i 0}, \theta\right)}{\partial \theta^{\prime}}-\left(\frac{1}{T} \sum_{t=1}^{T} \frac{\partial^{2} \log f\left(y_{i t} / \Omega_{i, t-1} ; a_{i 0}, \theta\right)}{\partial \theta \partial a_{i 0}}\right) Q_{i t}\left(\theta, a_{i 0}\right)\right] \\
& D_{i, \theta \theta, T}=\left[\frac{1}{T} \sum_{t=1}^{T} \frac{\partial^{2} \log f\left(y_{i t} / \Omega_{i, t-1} ; a_{i 0}, \theta\right)}{\partial \theta \partial \theta^{\prime}}\right]-\left[\frac{1}{T} \sum_{t=1}^{T} \frac{\partial^{2} \log f\left(y_{i t} / \Omega_{i, t-1} ; a_{i 0}, \theta\right)}{\partial \theta \partial a_{i 0}}\right]\left[\frac{1}{T} \sum_{t=1}^{T} \frac{\partial^{2} Q_{i t}\left(\theta, a_{i 0}\right)}{\partial \theta^{\prime}}\right] \\
& D_{\theta \theta, T}=\frac{1}{N} \sum_{i=1}^{N} D_{i, \theta \theta, T} .
\end{aligned}
$$

4) We assume that $\left(Y_{t, T}\right)$ obeys a central limit theorem i.e.

$$
\frac{1}{T} \sum_{t=1}^{T} Y_{t, T} \underline{d} N\left(0, I_{\theta \theta}\right)
$$

where $I_{\theta \theta}$ is assumed that has diagonal elements definite positive.

5) That as $T \rightarrow \infty ; D_{\theta \theta, T} \underline{d} D_{\theta \theta}>0$, where $D_{\theta \theta}$ is invertible. then

$$
\sqrt{T}(\hat{\theta}-\theta) \underline{d} N\left(0, D_{\theta \theta}^{-1} I_{\theta \theta} D_{\theta \theta}^{-1}\right) .
$$

Proof. The demonstration is well detailed in [13]. 


\section{Conclusion}

Through this work, we have tried to study, in the first part the fundamental probabilistic properties of BLGARCH (1, 2), basing on studies of Abdou Kâ Diongue, D. Guégan and R. C. Wolff [4] and G. Storti \& Vitale [1], that have been made in this class of models. In the second part, we have studied the statistical inference, extended the model on panel data structure, and used one of efficient method well called composite likelihood that was introduced by Lindsay [6]. This method has good properties under some general regularity conditions as the consistency property and the asymptotic normality of estimators.

\section{References}

[1] Storti, G. and Vitale, C. (2003) BL-GARCH Models and Asymmetries in Volatility. Statistical Methods and Applications, 12, 19-40. http://dx.doi.org/10.1007/BF02511581

[2] Engle, R.F. (1982) Autoregressive Conditional Heteroscedasticity with Estimates of the Variance of United Kingdom Inflation. Econometrica, 50, 987-1007. http://dx.doi.org/10.2307/1912773

[3] Bollerslev, T. (1986) Generalized Autoregressive Conditional Heteroskedasticity. Journal of Econometrics, 31, 307327. http://dx.doi.org/10.1016/0304-4076(86)90063-1

[4] Diongue, A.K., Guégan, D. and Wolff, R.C. (2009) BL-GARCH Models with Elliptical Distributed Innovations. Journal of Statistical Computation and Simulation, 80, 775-791. http://dx.doi.org/10.1080/00949650902773577

[5] Besag, J. (1974) Spatial Interaction and the Statistical Analysis of Latice Systems. Journal of the Royal Statistical Society: Series B, 36, 192-236.

[6] Lindsay, B. (1988) Composite Likelihood Methods. In: Prabhu, N.U., Ed., Statistical Inference from Stochastic Processes, American Mathematical Society, Providence, 221-239. http://dx.doi.org/10.1090/conm/080/999014

[7] Larribe, F. and Fearnhead, P. (2011) On Composite Likelihoods in Statistical Genetics. Statistica Sinica, 21, 43-69.

[8] Richard, A.D. and Chun, Y.Y. (2011) Comments on Pairwise Likelihood in Time Series Models. Statistica Sinica, 21, 255-277.

[9] Pakel, C., Shephard, N. and Sheppard, K. (2011) Nuisance Parameters, Composite Likelihoods and Panel of GARCH Models. Satistica Sinica, 21, 307-329.

[10] Monlenberghs, G. and Vervbeke, G. (2005) Models for Discrete Longitudinal Data. Springer, New York.

[11] Francq, C. and Zankoian, J.M. (2010) GARCH Models. Wiley, Hoboken. http://dx.doi.org/10.1002/9780470670057

[12] Engle, R.F. and Mezrich, J. (1996) GARCH for Groups. Risk, 9, 36-40.

[13] Engle, R.F., Shephard, N. and Sheppard, K. (2008) Fitting Vast Dimensional Time-Varying Covariance Models. Working Paper.

[14] Engle, R.F., Hendry, D.F. and Richard, J.F. (1983) Exogeneity. Econometrica, 51, 277-304. http://dx.doi.org/10.2307/1911990

[15] Wu, B., Yao, Q. and Zhu, S. (2013) Estimation in the Presence of Many Nuisance Parameters: Composite Likelihood and Plug-In Likelihood. Stochastic Processes and Their Applications, 123, 2877-2896. http://dx.doi.org/10.1016/j.spa.2013.03.017 
Scientific Research Publishing (SCIRP) is one of the largest Open Access journal publishers. It is currently publishing more than 200 open access, online, peer-reviewed journals covering a wide range of academic disciplines. SCIRP serves the worldwide academic communities and contributes to the progress and application of science with its publication.

Other selected journals from SCIRP are listed as below. Submit your manuscript to us via either submit@scirp.org or Online Submission Portal.
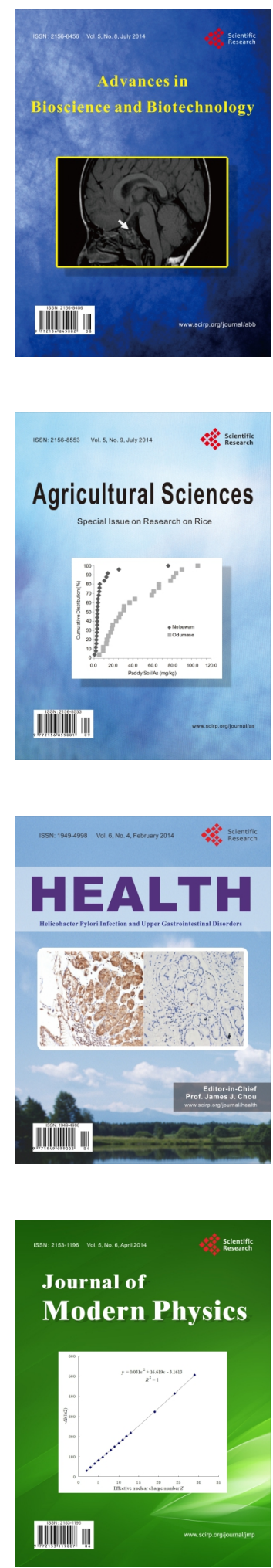
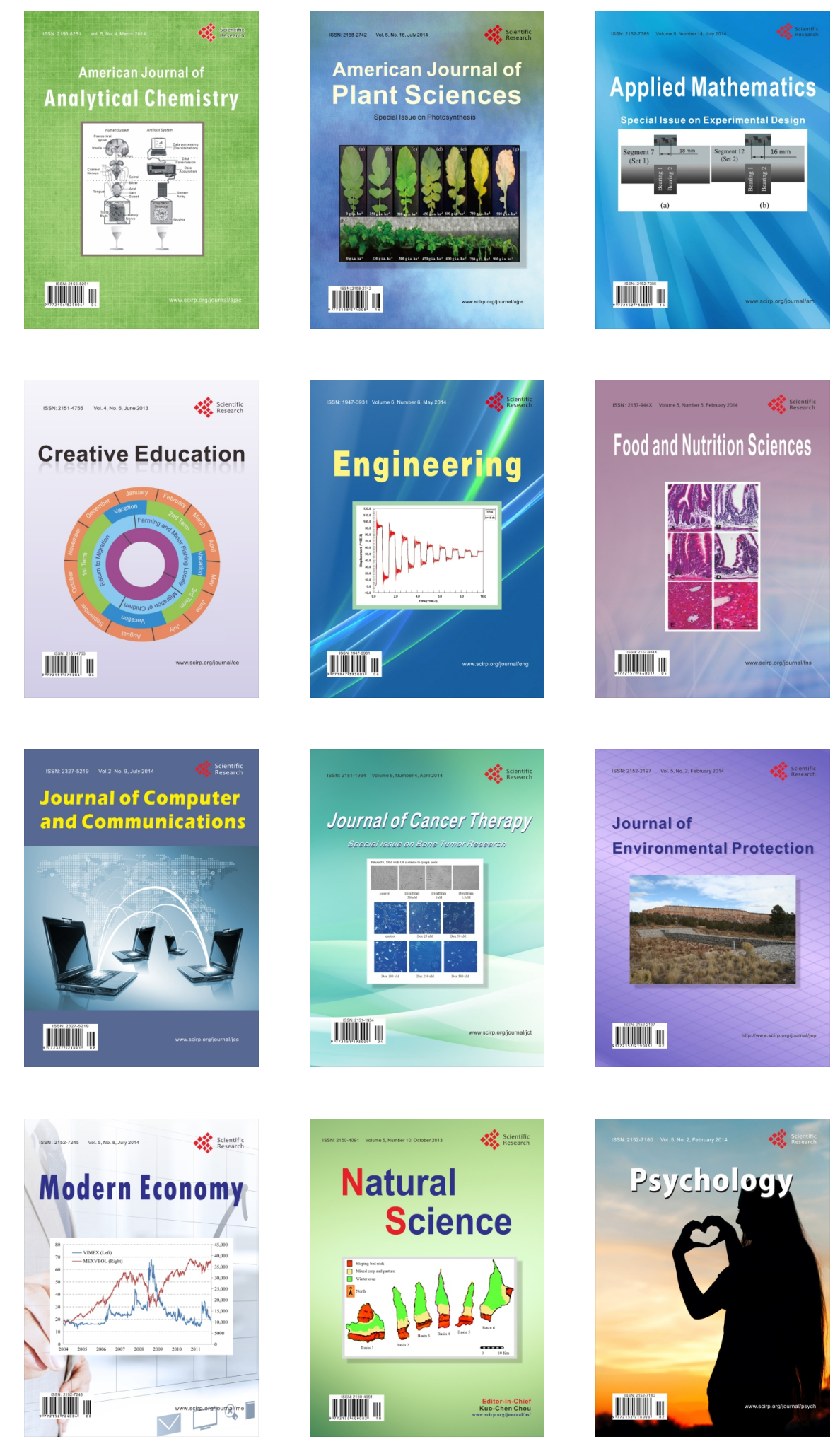